\title{
Relação entre habilidades auditivas e fonológicas em crianças com dislexia do desenvolvimento
}

\author{
Simone Aparecida Capellini \\ Giseli Donadon Germano \\ Ana Cláudia Vieira Cardoso
}

Habilidades auditiva-fonológica em disléxicos

\begin{abstract}
Resumo
A dislexia do desenvolvimento, dificuldade específica de leitura, é caracterizada pela dificuldade em realizar a decodificação fono-grafêmica e percepção de fonemas acusticamente semelhantes. Este estudo teve como objetivo caracterizar o desempenho de crianças com dislexia quanto às habilidades auditivas e de consciência fonológica, correlacionando-as. Participaram deste estudo crianças com dislexia e com bom desempenho escolar, submetidas a avaliações audiológica, do processamento auditivo e das habilidades fonológicas. Os resultados indicaram diferença estatisticamente significante entre as habilidades auditivas de seqüência para sons verbais, mensagem competitiva ipsi e contra-lateral, dicótico de dígitos e dissílabos alternados e ainda nos subtestes de síntese, segmentação, manipulação e transposição. Os achados deste estudo evidenciaram correlação entre provas de memória auditiva e manipulação silábica e fonêmica e associação entre habilidades auditivas e fonológicas, sugerindo que os processos auditivos interferem diretamente na percepção de aspectos acústicos, temporais e seqüenciais dos sons para formação de uma representação fonológica estável.
\end{abstract}

Palavras-chave: dislexia; consciência fonológica; desempenho.

\section{Relationship between phonological abilities and auditory processing in children with developmental dyslexia}

\begin{abstract}
Developmental dyslexia is a specific reading disability characterized by difficulty in phoneme-grapheme decoding and in perceiving similar pairs of similar phonemes. The aim of this study was to characterize the performance of dyslexic children in auditory and phonological awareness skills and correlate them on this population. A number of 20 children attending 2 nd to 4 th grades divided into GI: formed by 10 children with interdisciplinary diagnosis of dyslexia, both genders and aged 8 to 12 years old and GII: formed by 10 good readers, paired according to gender and age with GI. As procedure applied hearing evaluation, auditory processing evaluation and phonological awareness were used. The results revealed statistic significant difference between auditory skills of sequential memory for verbal sounds, speech intelligibility with ipsilateral and contralateral competitive message, dichotic of digits test, and staggered spondaic word test when the GI was compared to GII. The same occurred with the subtests of synthesis, segmentation, manipulation and transposition. The findings of this study evidenced correlation between hearing memory tests, syllabic and phonemic manipulation and association between hearing and phonologic abilities, suggesting that the hearing processes interfere directly in the acoustic aspects perception, temporal and sequential of the sounds for the formation of a stable phonologic representation.
\end{abstract}

Key words: dyslexia; phonological awareness; achievement.

\section{Relación entre habilidades auditivas y fonológicas en niños con dislexia del desarrollo}

\footnotetext{
Resumen

La dislexia del desarrollo, dificultad específica de lectura, se caracteriza por la dificultad en realizar la decodificación fono-grafema y percepción de fonemas acústicamente semejantes. Este estudio tuvo como objetivo caracterizar el desempeño de niños con dislexia en relación a las habilidades auditivas y de consciencia fonológica, correlacionándolas. Participaron de este estudio niños con dislexia y con buen desempeño escolar, sometidas a evaluaciones audio-lógicas, de procesamiento auditivo y de las habilidades fonológicas. Los resultados indicaron diferencia estadísticamente significativa entre las habilidades auditivas de secuencia para sonidos verbales, mensaje competitiva ipsi y contra lateral, dicótico de dígitos y disílabos alternados, y todavía, en los sub-testes de síntesis, segmentación, manipulación y transposición. Los hallazgos de este estudio muestran correlación entre pruebas de memoria auditiva y manipulación silábica y fonética y asociación entre habilidades auditivas y fonológicas, sugiriendo que los procesos auditivos interfieren directamente en la percepción de aspectos acústicos, temporales y secuenciales de los sonidos para formación de una representación fonológica estable. Palabras clave: dislexia; consciencia fonológica; performance.
} 


\section{Introdução}

A World Federation of Neurology, em 1968 (citado por Critchley 1985), definiu dislexia como sendo "transtorno de aprendizagem da leitura que ocorre, apesar de inteligência normal, de ausência de problemas sensoriais ou neurológicos, de instrução escolar adequada, de oportunidades sócioculturais suficientes, além disso, depende da existência de perturbação de aptidões cognitivas fundamentais, frequientemente de origem constitucional".

Nos últimos quarenta anos, pesquisadores têm defendido que a hipótese do déficit fonológico é um dos fatores da dislexia do desenvolvimento. Para esses pesquisadores, as crianças com tal diagnóstico apresentam dificuldades no uso da rota sublexical para a leitura, ou seja, no uso do mecanismo de conversão grafema-fonema em atividades que exigem habilidades fonológicas, como em leitura de palavras inventadas ou na categorização de palavras quanto aos sons (Bradley \& Bryant, 1983; Frith, 1985; Liberman \& Shankweiler, 1989; Olson, Wilse, Conners, \& Rack, 1990; Snowling, 1987; Stanovich, 1988).

Segundo Etchepareborda (2002) e ASHA (2003), as primeiras manifestações encontradas em crianças com dislexia aparecem na decodificação fonografêmica, quando a criança precisa entender e utilizar a associação dos sinais gráficos com as seqüências fonológicas das palavras no início da alfabetização.

As crianças com dislexia têm dificuldades em perceber pares de fonemas acusticamente semelhantes. A categorização do fonema é essencial para a percepção da fala, sendo que, nos bons leitores, as representações fonológicas estão categorizadas e armazenadas na memória de longa duração, formando o repertório básico com o qual a escrita pode ser associada (López-Escribano, 2007; Ortiz \& cols., 2007).

Dentre as falhas no processamento fonológico, encontram-se a dificuldade em realizar tarefas como a de análise, síntese, segmentação e omissão de fonemas. Assim, quando ocorrem alterações no desenvolvimento do processamento fonológico, as tarefas de identificação, localização e discriminação de fonemas, na palavra, encontram-se comprometidas (Iliadou \& Lakovides, 2003).

O processamento auditivo está diretamente relacionado à discriminação, memória e percepção auditiva. A discriminação auditiva é responsável por agrupar sons de acordo com a similaridade ou diferença, a memória auditiva é responsável por armazenar ou recuperar a informação auditiva, enquanto que a percepção auditiva é responsável por receber e interpretar os sons ou palavras recebidas. Tais competências são importantes na expressão e compreensão da palavra falada, na leitura, e na escrita (Dally, 2006; Kujala, Lovio, Lepisto, Laasonen, \& Näätänen, 2006; OlivaresGarcía \& cols., 2005).

As crianças disléxicas, por apresentarem déficit fonológico decorrente de uma carência no processamento temporal acústico, apresentam dificuldades quanto à discriminação, memória e percepção auditiva que comprometem diretamente o mecanismo de conversão letra-som, necessário 
para a realização da leitura e redação de textos em um sistema de escrita alfabético (Blomert, Mitterer, \& Paffen, 2004; Boets, Wouters, Wieringen \& Ghesquière, 2007; Moisescu-Yiflach, \& Pratt, 2005).

As habilidades fonológicas são necessárias para a leitura e a escrita, na medida em que a consciência fonológica será um aspecto a ser integrado no reconhecimento de palavras. A linguagem escrita deve ser considerada como um sistema de representação de língua, cuja aprendizagem significa a apropriação de um novo objeto de conhecimento. É necessário entender-se que a estrutura do sistema alfabético do português não significa que a escrita deste sistema seja a representação gráfica dos seus sons, mas sim que a percepção dos sons, durante a produção da linguagem oral, influencia diretamente o desenvolvimento da leitura e da escrita (Capellini \& Oliveira, 2003).

Nos últimos anos observou-se um crescente número de estudos que buscaram esclarecer a interrelação entre o desenvolvimento das habilidades do processamento auditivo e a aquisição das habilidades de consciência fonológica e, conseqüentemente, destas com o processo de aprendizagem da leitura e escrita (Cappovilla, \& Capovilla, 1998; Cherry \& Rubinstein, 2006; Mendonça \& Mendes, 2000; Moraes, 1996; Musiek, 1999; Sauer, 2005; Tallal \& cols., 1996).

Para o aprendizado inicial da leitura e escrita é necessária a percepção de informações acústicas para decodificar e codificar os fonemas. As crianças que apresentam dificuldades em processar os estímulos sonoros da fala poderão se deparar com obstáculos na segmentação e manipulação da estrutura fonológica da linguagem e, conseqüentemente, estarão sujeitas a apresentar dificuldades de leitura e escrita (Cherry \& Rubinstein, 2006; Doughterty, Cynader, Bjornson, Edgell \& Giaschi, 1998).

Com base no exposto acima, este trabalho teve por objetivo caracterizar o desempenho de escolares com dislexia quanto às habilidades auditivas e de consciência fonológica e relacionar as auditivas e fonológicas nesta população.

\section{Material e Método}

Este estudo foi realizado após aprovação do Comitê de Ética em Pesquisa da Universidade Estadual Paulista/UNESP sob o protocolo número 3118/2006.

Participaram deste estudo 20 escolares da $2^{\mathrm{a}}$ a $4^{\mathrm{a}}$ séries do ensino fundamental da rede pública municipal do município de Marília - São Paulo Brasil, distribuídos nos seguintes grupos:

Grupo I (GI): composto por 10 escolares da $2^{\text {a }}$ a $4^{\mathrm{a}}$ séries do ensino público municipal fundamental, com diagnóstico interdisciplinar de dislexia, sendo $60 \%$ do gênero masculino e $40 \%$ do gênero feminino, com média etária de 10 anos e 4 meses. Esses escolares foram submetidos à avaliação fonoaudiológica em um centro de estudos específico e, após a verificação dos sinais da dislexia, ou seja, presença de transtorno fonológico na fala, leitura e escrita, alterações em habilidades fonológicas e silábicas, presença de histórico familiar positivo para dislexia, os mesmos foram encaminhados para avaliação neurológica, neuropsicológica e de imagem num Ambulatório de 
Neurologia Infantil, onde o diagnóstico de dislexia do desenvolvimento foi confirmado. Os escolares foram considerados disléxicos quando apresentaram os seguintes critérios em situação de avaliação interdisciplinar: alteração quanto ao equilíbrio estático, à coordenação apendicular, persistência motora, equilíbrio dinâmico, coordenação troncomembro e sensibilidade no exame neurológico evolutivo, coeficiente intelectual normal, discrepância entre coeficiente intelectual verbal e execução na avaliação psicológica, alteração quanto à memória, leitura e escrita na bateria neuropsicológica, alterações fonêmicas, silábicas, rima e aliteração em provas de consciência fonológica, nível de leitura alfabético, velocidade de leitura oral abaixo do esperado para idade e escolaridade, transtorno fonológico evidenciado na avaliação fonológica, na leitura oral de textos e na leitura oral de palavras isoladas e na escrita sob ditado de palavras e pseudopalavras e na redação temática, compreensão parcial do texto lido. Como instrumentos de avaliação para o estabelecimento do perfil da dislexia foram utilizados: Exame Neurológico Evolutivo - ENE (Lefrève, 1976), Escala de Inteligência para Crianças - WISC III (Wechsler, 2002), Bateria Lúria-Nebraska - BLN (Ciasca, 1994), Avaliação Fonológica da Criança AFC (Yavas, Hernandorena \& Lamprecht, 1991), Prova de Consciência Fonológica - PCF (Capovilla \& Capovilla, 1998), Prova de Nomeação Automática Rápida - RAN (Capellini, Ferreira, Salgado \& Ciasca, 2007), Prova de Memória de Trabalho Fonológica (Capellini, Padula \& Ciasca, 2004), Prova de Leitura e Escrita (Pinheiro, 1994), Nível e Velocidade de Leitura Oral (Capellini \& Cavalheiro, 2000), Redação Temática.
Grupo II (GII): composto por 10 escolares da $2^{\mathrm{a}}$ a $4^{\mathrm{a}}$ séries do ensino fundamental, com bom desempenho escolar indicados pelas professoras a partir do desempenho satisfatório em dois bimestres consecutivos. Estes escolares foram pareados segundo gênero, média etária e escolaridade com o GI.

Os critérios de inclusão dos escolares nesse estudo foram: a assinatura do Termo de consentimento Livre e Esclarecido, escolares com acuidade visual, auditiva e desempenho cognitivo dentro dos padrões da normalidade, escolares com quadro de dislexia do desenvolvimento comprovada pela avaliação neurológica, neuropsicológica, fonoaudiológica e exame de imagem e nunca terem participado de programas de intervenção fonoaudiológica, pedagógica ou neuropsicológica. Enquanto os critérios de exclusão foram: a não apresentação da assinatura do Termo de Consentimento Livre e Esclarecido, escolares com diagnóstico interdisciplinar de Distúrbio de Aprendizagem, escolares que apresentassem acuidade visual e auditiva e desempenho cognitivo abaixo dos padrões da normalidade e outras síndromes genéticas ou neurológicas.

A coleta de dados foi realizada num centro de estudos de Educação e Saúde, em período contrário ao escolar. Conforme resolução do Conselho Nacional de Saúde CNS 196/96, anteriormente ao início das avaliações, os pais ou responsáveis dos pacientes selecionados assinaram o termo de Consentimento Pós-Informado para a autorização da realização do estudo.

$\mathrm{Na}$ avaliação da audição foram coletadas informações da sensibilidade auditiva dos escolares por meio de exame audiológico básico, constituído 
de audiometria tonal limiar, logoaudiometria e medidas de imitância acústica. Todos os participantes deste estudo apresentaram acuidade auditiva normal, ou seja, limiar auditivo de 20 dBNA. Posteriormente, todos os escolares foram submetidos à avaliação do processamento auditivo, utilizando-se as provas de Memória Seqüencial para Sons Não-Verbais (MSNV), Memória Seqüencial para Sons Verbais (MSV), Localização Sonora (LS) de Pereira $(1993,1996,1997)$, o teste de Logoaudiometria Pediátrica ou Teste de Inteligibilidade de Fala (PSI) com Mensagem Competitiva Contralateral (MCC) e Ipsilateral (MCI), o teste Dicótico de Dígitos e de Dissílabos Alternados - SSW (Almeida, Campos \& Almeida, 1988; Borges, 1997; Jerger, Jerger \& Hawkins, 1980; Santos \& Pereira, 1997; Zilioto, Kalil \& Almeida, 1997).

O teste de Memória Seqüencial para sons nãoverbais (MSNV) foi aplicado utilizando-se quatro instrumentos musicais: campânula vertical com cinco guizos, sino, coco e agogô pequeno. Apresentaram-se seqüências de quatro sons variando a ordem. Foram feitas demonstrações até que os escolares compreenderam sua tarefa. Inicialmente o escolar ficou sentado de frente para o instrumento para aprender. A testagem foi feita com o escolar de costas para o examinador e os instrumentos e depois virava de frente para desempenhar a tarefa. Foi considerado um bom desempenho identificar corretamente pelo menos duas seqüências de estímulos entre as três apresentadas (Cardoso, 1997).

O teste de Memória Sequencial Verbal (MSV) foi feito com as sílabas "pa", "ta" e "Ca", dispostas em três ordens diferentes. $\mathrm{O}$ escolar deveria repetir as sílabas na ordem em que elas foram apresentadas, sempre sentado de costas para o examinador. Considerou-se um bom desempenho a repetição correta de pelo menos duas das três ordens seqüenciais apresentadas (Cardoso, 1997).

O teste de localização sonora em cinco direções foi feito usando-se como estimulo o instrumento musical sino. O escolar foi instruído a apontar para a direção de onde ele acreditava originar o som. Avaliaram-se as posições à direita, à esquerda, acima, atrás e à frente da cabeça. Considerou-se resposta positiva quando o escolar localizava pelo menos quatro das cinco posições testadas (Cardoso, 1997).

O teste de Logoaudiometria Pediátrica ou Teste de Inteligibilidade de Fala (PSI) com Mensagem Competitiva Contralateral (MCC) e Ipsilateral (MCI), utiliza de dez sentenças as quais devem ser identificadas por meio de figuras. O escolar era posicionado dentro da cabine acústica de frente para uma prancha, contendo todas as figuras representantes das frases a serem ouvidas. A mensagem competitiva foi composta pela leitura de uma estória infantil (Pereira \& Schochat, 1997).

O teste Dicótico de Dígitos utiliza de uma lista de 20 pares de dígitos, sendo que 4 dígitos são apresentados dicoticamente ao sujeito, que deve repetir oralmente todos os dígitos apresentados. A lista é apresentada novamente mais duas vezes, sendo que, na segunda apresentação, o indivíduo deve repetir os dígitos ditos na orelha direita e, na terceira vez, na orelha esquerda. Essas etapas são denominadas escuta direcionada para a direita e 
Tabela 1. Distribuição das médias, desvios-padrão e significância $(p)$ do desempenho dos escolares dos GI e GII nas Provas Auditivas.

\begin{tabular}{|c|c|c|c|c|c|}
\hline Instrumentos & & Grupo & Média & Desvio-padrão & Significância $(p)$ \\
\hline \multirow{3}{*}{$\begin{array}{l}\text { Prova de Memória } \\
\text { Seqüencial } \\
\text { Localização Sonora }\end{array}$} & \multirow[t]{2}{*}{ MSNV } & I & 0,53 & 0,28 & \multirow{2}{*}{$0,146^{*}$} \\
\hline & & II & 0,73 & 0,31 & \\
\hline & \multirow[t]{2}{*}{ MSV } & I & 0,48 & 0,34 & \multirow{2}{*}{$0,014 *$} \\
\hline & & II & 0,88 & 0,32 & \\
\hline & \multirow[t]{2}{*}{ LS } & $\mathrm{I}$ & 0,74 & 0,35 & \multirow{2}{*}{$0,349^{*}$} \\
\hline & & II & 0,86 & 0,16 & \\
\hline \multirow{17}{*}{$\begin{array}{l}\text { Teste } \\
\text { Inteligibilidade } \\
\text { Fala }\end{array}$} & \multirow{2}{*}{$\begin{array}{l}\text { MCC- } \\
40 D\end{array}$} & I & 89.00 & 7.38 & \multirow{2}{*}{$0,010^{*}$} \\
\hline & & II & 97,00 & 4,83 & \\
\hline & \multirow{3}{*}{$\begin{array}{l}\text { MCC- } \\
40 E\end{array}$} & I & 94.00 & 9.66 & \multirow{3}{*}{$0,246^{*}$} \\
\hline & & & & & \\
\hline & & II & 98,00 & 4,22 & \\
\hline & \multirow[t]{2}{*}{ MCI0D } & I & 89,00 & 15,24 & \multirow{2}{*}{$0,176^{*}$} \\
\hline & & II & 97,00 & 9,49 & \\
\hline & \multirow[t]{2}{*}{ MCI-10D } & I & 75,00 & 19,00 & \multirow{2}{*}{$0,004 *$} \\
\hline & & II & 98,00 & 4,22 & \\
\hline & \multirow[t]{2}{*}{ MCI-15D } & I & 78,00 & 16,19 & \multirow{2}{*}{$0,005^{*}$} \\
\hline & & II & 96,00 & 6,99 & \\
\hline & \multirow[t]{2}{*}{ MCIOE } & I & 94,00 & 8,43 & \multirow{2}{*}{$0,051^{*}$} \\
\hline & & II & 100,00 & 0,00 & \\
\hline & \multirow[t]{2}{*}{ MCI-10E } & I & 82,00 & 21,50 & \multirow{2}{*}{$0,027 *$} \\
\hline & & II & 100,00 & 0,00 & \\
\hline & \multirow[t]{2}{*}{ MCI-15E } & I & 76,00 & 12,65 & \multirow{2}{*}{$<0,001^{*}$} \\
\hline & & II & 99,00 & 3,16 & \\
\hline \multirow[t]{8}{*}{ Dicótico de Dígitos } & \multirow[t]{2}{*}{ IntBinOD } & I & 55,50 & 14,06 & $<0001 *$ \\
\hline & & II & 89,88 & 8,40 & $<0,001^{*}$ \\
\hline & IntBinOE & I & 55,25 & 13,79 & $<0001 *$ \\
\hline & & II & 87,75 & 6,31 & 0,001 \\
\hline & Direita & I & 69,50 & 16,28 & \\
\hline & & II & 92,00 & 10,79 & $0,002^{*}$ \\
\hline & Esquerda & I & 53,50 & 20,30 & \\
\hline & & II & 87,13 & 19,82 & $0,001^{*}$ \\
\hline Teste Dissílabos & OD & I & 68,38 & 14,31 & $0007 *$ \\
\hline Alternados & & II & 87,00 & 13,18 & 0,007 \\
\hline & $\mathrm{OE}$ & I & 58,48 & 12,66 & $<\cap \Omega \cap 1$ \\
\hline & & II & 86,63 & 8,52 & $<0,001 *$ \\
\hline Índice & OD & I & 95,60 & 3,50 & 0616 \\
\hline Reconhecimento de & & II & 96,40 & 3,50 & $0,010^{*}$ \\
\hline Fala & $\mathrm{OE}$ & I & 95,20 & 3,16 & $0051 *$ \\
\hline & & II & 98,00 & 2,83 & 0,051 \\
\hline
\end{tabular}

Legenda: Memória Seqüencial para Sons Não-verbais: MSNV; Memória Seqüencial para Sons Verbais: MSV; Localização Sonora: LS; Mensagem Competitiva Contralateral: MCC; Mensagem Competitiva Ipsilateral: MCI; Integração binaural: IntBin; Orelha Direita: OD; Orelha Esquerda: OE. 
para a esquerda. Os resultados obtidos foram registrados em uma folha de marcação que contém dados do teste. Foi considerado erro quando o indivíduo faz omissão ou substituição do dígito. Os resultados foram analisados segundo critério de Pereira e Schochat (1997), sendo padrão de normalidade $10 \%$ de erros, indicando uma boa habilidade para agrupar componentes do sinal acústico em figura-fundo e identificá-los verbalmente. Os resultados alterados em ambas as orelhas sugerem alterações no hemisfério esquerdo.

O teste Dissílabos Alternados é apresentado numa intensidade $50 \mathrm{~dB}$ NS e contém 40 itens, sendo que cada item é formado por 4 palavras compostas por 2 pares de dissílabos paroxítonos (por ex.: bota/ fora/ pega/ fogo). Cada palavra é apresentada a cada orelha, havendo uma superposição parcial, isto é, a segunda sílaba da palavra inicial e a primeira sílaba da palavra final são apresentadas simultaneamente às duas orelhas.

$\mathrm{O}$ procedimento utilizado para avaliação das habilidades fonológicas foi a Prova de Consciência Fonológica desenvolvida por (Capovilla \& Capovilla, 1998). Esta prova é composta de 10 subtestes, com 4 itens cada, que se referem à verificação das habilidades fonêmicas, suprafonêmicas e silábicas. O resultado desta prova é apresentado em forma de escore para os subtestes e escore total baseado no número de erros.

Com o intuito de verificarem-se possíveis diferenças entre as médias das variáveis de interesse, aplicou-se o Teste $t$ de Student, controlado pelo Teste de Levene para Igualdade de Variâncias. Para serem verificadas as relações entre os pares de variáveis (os testes SSW, de Inteligibilidade de Fala (PSI), MSNV e MSV, e as Provas de Consciência Fonológica) utilizou-se a Análise de Correlação de Spearman. Para a aplicação dos testes estatísticos deste estudo, adotamos o nível de 5\% de significância marcada com asterisco. Para análise dos dados, foi utilizado o programa SPSS (Statistical Package for Social Sciences), em sua versão 13.0.

\section{Resultados}

Na Tabela 1, apresentamos o desempenho dos escolares do GI e GII na provas auditivas.

Os resultados analisados pelo Teste t de Student, controlado pelo Teste de Levene para Igualdade de Variâncias, evidenciaram diferença significante para o desempenho dos escolares do GI e GII na prova de memória seqüencial para sons verbais (MSV), demonstrando melhor desempenho dos escolares do GII em relação ao GI.

Os resultados analisados pelo Teste $\mathrm{t}$ de Student, controlado pelo Teste de Levene para Igualdade de Variâncias do Teste de Inteligibilidade de Fala (PSI) com Mensagem Competitiva Contralateral (MCC) e Ipsilateral (MCI) evidenciaram diferença significante para o desempenho dos escolares do GI e GII com MCC direita para as relações fala/competição -10, -15 e -40 e MCI esquerda para a relação fala/competição -10 , ressaltando melhor desempenho dos escolares do GII em relação ao GI.

No Teste de Dicótico de Dígitos observamos diferença significante para o desempenho dos escolares do GI e GII nas etapas de integração binaural (direita e esquerda) e separação binaural 
Tabela 2. Distribuição das médias do números de erros, desvios-padrão e significância ( $p)$ do desempenho dos escolares dos GI e GII da Prova de Consciência Fonológica (PCF).

\begin{tabular}{|c|c|c|c|c|}
\hline Habilidades & Grupo & Média & Desvio-padrão & Significância $(p)$ \\
\hline \multirow[t]{2}{*}{$\mathrm{SiS}$} & I & 0,20 & 0,42 & \multirow{2}{*}{$0,556^{*}$} \\
\hline & II & 0,10 & 0,32 & \\
\hline \multirow[t]{2}{*}{$\mathrm{SiF}$} & I & 3,00 & 1,05 & \multirow{2}{*}{$0,001^{*}$} \\
\hline & II & 1,10 & 0,99 & \\
\hline \multirow[t]{2}{*}{ Rim } & I & 2,80 & 0,92 & \multirow{2}{*}{$<0,001 *$} \\
\hline & II & 0,20 & 0,42 & \\
\hline \multirow[t]{2}{*}{ Ali } & I & 1,60 & 1,07 & \multirow{2}{*}{$0,002^{*}$} \\
\hline & II & 0,20 & 0,42 & \\
\hline \multirow[t]{2}{*}{ Ses } & I & 0,10 & 0,32 & \multirow{2}{*}{$0,343 *$} \\
\hline & II & 0,00 & 0,00 & \\
\hline \multirow[t]{2}{*}{$\mathrm{SeF}$} & I & 2,80 & 1,23 & \multirow{2}{*}{$<0,001 *$} \\
\hline & II & 0,40 & 0,52 & \\
\hline \multirow[t]{2}{*}{ ManS } & I & 3,00 & 1,05 & \multirow{2}{*}{$<0,001 *$} \\
\hline & II & 0,30 & 0,48 & \\
\hline \multirow[t]{2}{*}{ ManF } & I & 3,70 & 0,48 & \multirow{2}{*}{$<0,001^{*}$} \\
\hline & II & 0,50 & 0,53 & \\
\hline \multirow[t]{2}{*}{ TranS } & I & 2,60 & 1,51 & \multirow{2}{*}{$0,001 *$} \\
\hline & II & 0,20 & 0,42 & \\
\hline \multirow[t]{2}{*}{ TranF } & I & 3,70 & 0,95 & \multirow{2}{*}{$0,175^{*}$} \\
\hline & II & 3,00 & 1,25 & \\
\hline \multirow[t]{2}{*}{ ET } & I & 23,50 & 5,10 & \multirow{2}{*}{$<0,001^{*}$} \\
\hline & II & 6,00 & 1,56 & \\
\hline
\end{tabular}

Legenda: Síntese Silábica: SiS; Síntese Fonêmica: SiF; Rima: Rim; Aliteração: Ali; Segmentação Silábica: SeS; Segmentação Fonêmica: SeF; Manipulação Silábica: ManS; Manipulação Fonêmica: ManF; Transposição Silábica: TranS; Transposição Fonêmica: TranF; Escore Total: ET.

(atenção direita e atenção esquerda), corroborando melhor desempenho dos escolares do GII em relação ao GI.

No Teste de Dissílabos Alternados (SSW) verificamos diferença significante para o desempenho dos escolares do GI e GII para orelha direita e esquerda, o que mostrou um melhor desempenho dos escolares do GII em relação ao GI.
Não ocorreu diferença significante para o desempenho dos escolares do GII e GI, tendo demonstrado que a atuação dos dois grupos foi semelhante no Índice de Reconhecimento de Fala.

Entre as provas de processamento auditivo, não foram observadas correlações estatisticamente significantes entre as variáveis na comparação intragrupo. 
Tabela 3. Distribuição dos níveis de significância (p) do desempenho dos GI e GII, em função da comparação dos subtestes silábicos e fonêmicos da prova de consciência fonológica.

\begin{tabular}{lrr}
\hline \multicolumn{1}{c}{ Habilidades } & GI & \multicolumn{1}{c}{ GII } \\
\hline SiS x SiF & $<0,001^{*}$ & $0,015^{*}$ \\
Rim x Ali & $0,058^{*}$ & $>0,999^{*}$ \\
SeS x SeF & $<0,001^{*}$ & $0,037^{*}$ \\
Mans x Manf & $0,045^{*}$ & $0,443^{*}$ \\
TranS x TranF & $0,017^{*}$ & $<0,001^{*}$ \\
\hline
\end{tabular}

Legenda: Síntese Silábica: SiS; Síntese Fonêmica: SiF; Rima: Rim; Aliteração: Ali; Segmentação Silábica: SeS; Segmentação Fonêmica: SeF; Manipulação Silábica: ManS; Manipulação Fonêmica: ManF; Transposição Silábica: TranS; Transposição Fonêmica: TranF.

Tabela 4. Medidas de análise de relação entre o Teste de Dissílabos Alternados (SSW), a Síntese fonêmica (SiF), Síntese silábica (SiS), Segmentação fonêmica (SeF) e Segmentação silábica (SeS).

\begin{tabular}{|c|c|c|c|c|c|c|c|}
\hline \multirow{2}{*}{ SSW } & & \multicolumn{2}{|c|}{ Todos } & \multicolumn{2}{|c|}{ Grupo I } & \multicolumn{2}{|c|}{ Grupo II } \\
\hline & & OD & $\mathrm{OE}$ & OD & $\mathrm{OE}$ & OD & $\mathrm{OE}$ \\
\hline \multirow[t]{3}{*}{$\mathrm{SiS}$} & Coeficiente de Correlação & $-0,05$ & 0,07 & $-0,22$ & 0,44 & 0,42 & 0,23 \\
\hline & Nível de significância $(p)$ & 0,838 & 0,760 & 0,543 & 0,209 & 0,228 & 0,517 \\
\hline & $\mathrm{N}$ & 20 & 20 & 10 & 10 & 10 & 10 \\
\hline \multirow[t]{3}{*}{$\mathrm{SiF}$} & Coeficiente de Correlação & $-0,74$ & $-0,63$ & $-0,60$ & 0,24 & $-0,32$ & $-0,28$ \\
\hline & Nível de significância $(p)$ & $0,000^{*}$ & $0,003 *$ & 0,069 & 0,497 & 0,376 & 0,432 \\
\hline & $\mathrm{N}$ & 20 & 20 & 10 & 10 & 10 & 10 \\
\hline \multirow[t]{3}{*}{$\mathrm{SeS}$} & Coeficiente de Correlação & $-0,18$ & $-0,14$ & $-0,06$ & 0,17 & - & - \\
\hline & Nível de significância $(p)$ & 0,448 & 0,558 & 0,873 & 0,631 & - & - \\
\hline & $\mathrm{N}$ & 20 & 20 & 10 & 10 & - & - \\
\hline \multirow[t]{3}{*}{$\mathrm{SeF}$} & Coeficiente de Correlação & $-0,46$ & $-0,52$ & $-0,17$ & 0,44 & 0,40 & 0,43 \\
\hline & Nível de significância $(p)$ & $0,040^{*}$ & $0,019 *$ & 0,648 & 0,201 & 0,248 & 0,218 \\
\hline & $\mathrm{N}$ & 20 & 20 & 10 & 10 & 10 & 10 \\
\hline
\end{tabular}

Na Tabela 2, apresentamos o desempenho dos escolares do GI e GII na prova de consciência fonológica (PCF). Os resultados analisados pelo Teste t de Student, controlado pelo Teste de Levene para Igualdade de Variâncias, comprovaram diferença significante para o desempenho dos escolares do GI e GII nos subtestes de síntese fonêmica $(\mathrm{SiF})$, segmentação fonêmica $(\mathrm{SeF})$, rima (RIM), aliteração (ALI), manipulação silábica (ManS), manipulação fonêmica (ManF), 
Tabela 5. Medidas de análise de relação entre o Teste de Inteligibilidade de Fala (PSI) e o de Mensagem Competitiva Contralateral (MCC), Mensagem Competitiva Ipsilateral (MCI), Rima (Rim) e Aliteração (Alit).

\begin{tabular}{|c|c|c|c|c|c|c|c|}
\hline \multirow{2}{*}{ PSI } & & \multicolumn{2}{|c|}{ Todos } & \multicolumn{2}{|c|}{ Grupo I } & \multicolumn{2}{|c|}{ Grupo II } \\
\hline & & Rim & Alit & Rim & Alit & Rim & Alit \\
\hline \multirow[t]{3}{*}{ MCC-40D } & Coeficiente de Correlação & $-0,45$ & $-0,40$ & $-0,04$ & 0,02 & 0,33 & 0,33 \\
\hline & Nível de significância $(p)$ & $0,049^{*}$ & 0,083 & 0,923 & 0,955 & 0,356 & 0,356 \\
\hline & $\mathrm{N}$ & 20 & 20 & 10 & 10 & 10 & 10 \\
\hline \multirow[t]{3}{*}{ MCC-40E } & Coeficiente de Correlação & $-0,28$ & $-0,09$ & $-0,38$ & $-0,05$ & 0,25 & 0,25 \\
\hline & Nível de significância $(p)$ & 0,226 & 0,696 & 0,274 & 0,890 & 0,486 & 0,486 \\
\hline & $\mathrm{N}$ & 20 & 20 & 10 & 10 & 10 & 10 \\
\hline \multirow[t]{3}{*}{ MCC-IOD } & Coeficiente de Correlação & $-0,49$ & $-0,31$ & 0,07 & 0,21 & $-0,67$ & $-0,67$ \\
\hline & Nível de significância $(p)$ & $0,030 *$ & 0,187 & 0,849 & 0,563 & $0,035^{*}$ & $0,035^{*}$ \\
\hline & $\mathrm{N}$ & 20 & 20 & 10 & 10 & 10 & 10 \\
\hline \multirow[t]{3}{*}{ MCI-10D } & Coeficiente de Correlação & $-0,75$ & $-0,46$ & 0,142 & 0,54 & $-0,38$ & $-0,38$ \\
\hline & Nível de significância $(p)$ & $0,000 *$ & $0,040^{*}$ & 0,696 & 0,104 & 0,286 & 0,286 \\
\hline & $\mathrm{N}$ & 20 & 20 & 10 & 10 & 10 & 10 \\
\hline \multirow[t]{3}{*}{ MCI-15D } & Coeficiente de Correlação & $-0,51$ & $-0,57$ & 0,39 & $-0,11$ & $-0,32$ & $-0,32$ \\
\hline & Nível de significância $(p)$ & $0,022 *$ & $0,008 *$ & 0,264 & 0,764 & 0,363 & 0,363 \\
\hline & $\mathrm{N}$ & 20 & 20 & 10 & 10 & 10 & 10 \\
\hline \multirow[t]{3}{*}{ MCI-10E } & Coeficiente de Correlação & $-0,57$ & $-0,26$ & $-0,36$ & 0,14 & - & - \\
\hline & Nível de significância $(p)$ & $0,009^{*}$ & 0,277 & 0,314 & 0,693 & - & - \\
\hline & $\mathrm{N}$ & 20 & 20 & 10 & 10 & - & - \\
\hline \multirow[t]{3}{*}{ MCI-10E } & Coeficiente de Correlação & $-0,65$ & $-0,32$ & $-0,03$ & 0,25 & - & - \\
\hline & Nível de significância $(p)$ & $0,002 *$ & 0,164 & 0,927 & 0,480 & - & - \\
\hline & $\mathrm{N}$ & 20 & 20 & 10 & 10 & - & - \\
\hline \multirow[t]{3}{*}{ MCI-15E } & Coeficiente de Correlação & $-0,80$ & $-0,49$ & $-0,24$ & 0,39 & 0,17 & 0,17 \\
\hline & Nível de significância $(p)$ & $0,000^{*}$ & $0,030^{*}$ & 0,510 & 0,261 & 0,645 & 0,645 \\
\hline & $\mathrm{N}$ & 20 & 20 & 10 & 10 & 10 & 10 \\
\hline
\end{tabular}

transposição silábica (TranS) e no escore total (ET), ratificando melhor desempenho dos escolares do GII em relação ao GI.

Comparando o desempenho dos escolares do GI e GII quanto às provas silábicas, fonêmicas e suprafonêmicas da prova de consciência fonológica, verificamos, nos resultados analisados pelo Teste $t$ de Student para Dados Pareados, diferença estatisticamente significante nos subtestes de síntese, segmentação, manipulação e transposição, provando melhor desempenho dos escolares de ambos os grupos nas provas silábicas do que nas fonêmicas (Tabela 3).A Tabela 4 apresenta a análise da relação entre o Teste de Dissílabos Alternados 
Tabela 6. Medidas de análise de relação entre as Provas de Memória Seqüencial para Sons Não-verbais, Memória Sequiencial para Sons Verbais, Manipulação silábica, Manipulação Fonêmica, Transposição Silábica e Transposição Fonêmica.

\begin{tabular}{|c|c|c|c|c|c|c|c|}
\hline & \multicolumn{2}{|c|}{ Todos } & \multicolumn{2}{|c|}{ Grupo I } & \multicolumn{2}{|c|}{ Grupo II } \\
\hline & & MSNV & MSV & MSNV & MSV & MSNV & MSV \\
\hline \multirow[t]{3}{*}{ ManS } & Coeficiente de Correlação & $-0,45$ & $-0,47$ & $-0,24$ & $-0,04$ & $-0,38$ & 0,33 \\
\hline & Nível de significância $(p)$ & $0,046^{*}$ & $0,035^{*}$ & 0,503 & 0,922 & 0,282 & 0,359 \\
\hline & $\mathrm{N}$ & 20 & 20 & 10 & 10 & 10 & 10 \\
\hline \multirow[t]{3}{*}{ ManF } & Coeficiente de Correlação & $-0,33$ & $-0,65$ & $-0,04$ & $-0,04$ & 0,23 & $-0,50$ \\
\hline & Nível de significância $(p)$ & 0,158 & $0,002 *$ & 0,910 & 0,915 & 0,521 & 0,144 \\
\hline & $\mathrm{N}$ & 20 & 20 & 10 & 10 & 10 & 10 \\
\hline \multirow[t]{3}{*}{ TranS } & Coeficiente de Correlação & $-0,29$ & $-0,53$ & $-0,12$ & $-0,09$ & 0,14 & $-0,31$ \\
\hline & Nível de significância $(p)$ & 0,212 & $0,017 *$ & 0,736 & 0,811 & 0,691 & 0,382 \\
\hline & $\mathrm{N}$ & 20 & 20 & 10 & 10 & 10 & 10 \\
\hline \multirow[t]{3}{*}{ TranF } & Coeficiente de Correlação & $-0,03$ & $-0,07$ & $-0,19$ & 0,24 & 0,37 & 0,20 \\
\hline & Nível de significância $(p)$ & 0,890 & 0,763 & 0,602 & 0,509 & 0,297 & 0,580 \\
\hline & $\mathrm{N}$ & 20 & 20 & 10 & 10 & 10 & 10 \\
\hline
\end{tabular}

Legenda: Provas de Memória Seqüencial para Sons Não-verbais: MSNV; Memória Seqüencial para Sons Verbais: MSV; Manipulação silábica: ManS; Manipulação Fonêmica: ManF; Transposição Silábica: TranS; Transposição Fonêmica: TranF.

(SSW), Síntese fonêmica e silábica ( $\mathrm{SiF}, \mathrm{SiS})$ e Segmentação fonêmica e silábica ( $\mathrm{SeF}, \mathrm{SeS}$ ), uma vez que, para a realização da análise dos segmentos acústicos da palavra, é necessária a organização dos espectros acústicos para a percepção da fala.

Os resultados revelaram correlação estatisticamente significante para a comparação entre síntese e segmentação fonêmica nas orelhas direita e esquerda, para todos os integrantes dos grupos. A Tabela 5 apresenta a análise da relação entre o Teste de Inteligibilidade de Fala (PSI) com Mensagem Competitiva Contralateral (MCC) e Ipsilateral (MCI), e Rima (Rim) e Aliteração (Alit), uma vez que, para a realização da análise dos mesmos segmentos silábicos ou fonêmicos em uma seqüência de palavras, é necessária atenção na codificação e percepção dos segmentos, para identificação de semelhanças de amostras na fala.
Os resultados revelaram correlação estatisticamente significante para a comparação entre rima e mensagem competitiva contralateral na orelha direita e mensagem competitiva ipsilateral na orelha esquerda para todos os integrantes dos grupos; entre rima, aliteração e mensagem competitiva ipsilateral direita e esquerda para todos os grupos e para o GII .

A Tabela 6 apresenta a análise da relação entre as Provas de Memória Seqüencial para Sons NãoVerbais (MSNV), Memória Seqüencial para Sons Verbais (MSV), Manipulação silábica (ManS), Manipulação Fonêmica (ManF), Transposição Silábica (TranS) e Transposição Fonêmica (TranF), visto que, para acionar o mecanismos gerativos de sons e sílabas para a formação de novas palavras, é necessária atenção para a codificação e uso de integração e memória na manipulação dos segmentos da fala. 
Os resultados revelaram correlação estatisticamente significante para a comparação entre MSNV e manipulação silábica, e entre MSV e manipulação silábica, fonêmica e transposição silábica para todos os integrantes dos grupos.

\section{Discussão}

Os achados deste estudo revelaram que os escolares com dislexia do desenvolvimento apresentam dificuldade em lidar com informações fonológicas na memória, necessárias para a aprendizagem da leitura (Bradley \& Bryant, 1983; Dally, 2006; Frith, 1985; López-Escribano, 2007; Olivares-García \& cols., 2005; Ortiz \& cols., 2007; Snowling, 1987).

O desempenho dos escolares do GI nas habilidades auditivas revelou presença de dificuldade na organização de eventos sonoros, dificuldade na memória auditiva de curta duração e dificuldade em perceber sons competitivos (Dawes, Bishop, Sirimanna \& Bamiou, 2008; Tallal \& Gaab 2008). Considerando que, para a realização da leitura, é necessária a lembrança temporal acústica da ordem dos fonemas para evocar a ordem espacial dos grafemas, os escolares com dislexia apresentaram considerável dificuldade para a realização da correspondência fonema-grafema, tarefa esta realizada em grande parte pela memória seqüencial auditiva, que como vimos neste estudo se correlaciona com manipulação e transposição, habilidades necessárias para composição e formação de palavras e novas palavras.

A categorização do fonema é essencial para a percepção da fala, sendo que, nos bons leitores, as representações fonológicas estão categorizadas e armazenadas na memória de longa duração, formando o repertório básico com o qual a escrita pode ser associada (Iliadou \& Lakovides, 2003; López-Escribano, 2007). Como evidenciado neste estudo, os escolares com dislexia apresentam dificuldades quanto a esta categorização, constatadas pelo desempenho inferior em tarefas de rima e aliteração se comparado ao grupo de bons leitores, denotando que a atenção, discriminação e percepção de segmentos semelhantes na palavra encontram-se comprometidas.

$\mathrm{Na}$ tarefa de dicótico de dígitos, os escolares com dislexia apresentaram desempenho inferior, demonstrando que a presença de hipoperfusão na região mesial do lobo temporal pode influenciar a percepção para sequencialização dos sons durante a leitura. Estes resultados vão de encontro aos descritos na literatura nacional (Arduini, Capellini \& Ciasca, 2006; Sauer, Pereira, Ciasca, Pestun \& Guerreiro, 2006).

O desempenho dos escolares em consciência fonológica revelou que os escolares do GII apresentaram melhor desempenho nas habilidades silábicas e fonêmicas para segmentação, manipulação, transposição, rima e aliteração do que os escolares do GI. Entretanto, verificou-se, também, que tanto nos escolares do GI, como nos do GII ocorreu um melhor desempenho nas habilidades silábicas do que nas fonêmicas, evidenciando que esta dificuldade para perceber estruturas fonêmicas não é característica única de escolares com dislexia do desenvolvimento, mas sim uma alteração que pode decorrer do ensino de base do sistema de escrita na língua portuguesa. 
Neste estudo, pelo fato da escola utilizar metodologia de alfabetização baseada na filosofia construtivista, verificou-se que, quando não são oferecidas instruções diretas sobre a correspondência letra-som, necessária para a aprendizagem do princípio alfabético, tanto escolares com dislexia do desenvolvimento, como escolares sem problemas de aprendizagem apresentam falhas em mecanismos gerativos para a formação de palavras, ou seja, a capacidade de reter informações na memória de trabalho e de manipulálas para formação de novas palavras a partir de um segmento (som ou sílaba).

A percepção da estrutura fonêmica da fala permite que a criança utilize um sistema gerativo para converter ortografia em fonologia, permitindo, assim, a leitura de qualquer palavra regular que envolva a correspondência grafofonêmica (Blomert \& cols., 2004; Boets \& cols., 2007; Capellini \& Oliveira, 2003; López-Escribano, 2007; MoisescuYiflach \& Pratt, 2005; Ortiz \& cols., 2007).

A característica gerativa das ortografias alfabéticas permite aos leitores aprenderem por si mesmos, uma vez que, ao encontrar palavras novas, eles possam aplicar as regras de decodificação fonológica. O processo de decodificação fonológica contribui para que a criança forme a representação ortográfica da nova palavra, admitindo, dessa forma, que a palavra nova seja lida pela rota lexical. Portanto, é o processo fonológico que permitirá que a criança, posteriormente, leia pela rota lexical (Alégria \& Mousty, 1996; Capellini, 2004; Kujala \& cols., 2006; López-Escribano, 2007).

Neste estudo, observou-se que os alunos do GI apresentam dificuldades em habilidades auditivas que comprometem a codificação e organização dos espectros acústicos, o que prejudica o uso de habilidades fonológicas como as de análise e síntese do material verbal e de leitura, conforme descritas por Byrne \& Fielding-Barnsley, (1991), Gregoire \& Pierart (1997), Ratner, Gleason, Narasimhan (1999) e Ávila (2004), Olivares-García e colaboradores (2005), Dally (2006) e Kujala e colaboradores (2006).

\section{Considerações Finais}

Os achados deste estudo permitiram concluir que os escolares com dislexia do desenvolvimento apresentam dificuldades em habilidades auditivas de atenção, codificação, organização e integração de informações auditivas que comprometem o uso de habilidades fonológicas como a atenção, análise, síntese e memória de trabalho.

Há associação entre habilidades auditivas e habilidades fonológicas significantes que sugerem que os processos auditivos interferem diretamente na percepção de aspectos acústicos, temporais e seqüenciais dos sons para a formação de uma representação fonológica estável.

A realização deste estudo pode apresentar algumas limitações, seja pelo número de sujeitos ou pelas correlações estabelecidas, em decorrência disto, tornam-se necessários estudos complementares que investiguem as correlações aqui evidenciadas em um maior número de escolares a fim de estabelecer um aprofundamento científico na relação existente entre consciência fonológica, percepção e processamento auditivo, uma vez que não há muitos estudos na literatura nacional. 


\section{Referências}

Alégria, J., \& Mousty, P. (1996). The development of spelling procedures in French- speaking, normal and reading-disabled children: effects so frequency and lexicality. Journal of experimental child psychology, 63, 312-338.

Almeida, C. R. I., Campos, M. I., \& Almeida, R. R. (1988). Logoaudiometria Pediátrica (PSI). Revista Brasileira de Otorrinolaringologia, 54(3), 73-6.

Arduini, R., Capellini, S. A., \& Ciasca, S. M. (2006). Comparative study neuropsychological and neuroimaging evaluations in children with dyslexia. Arquivos de Neuro-psiquiatria, 64(2b), 369-375.

ASHA (2003). Language-Based Learning Disabilities [On-line]. Recuperado: 12 out. 2003. Disponível: http://www.asha.org

Ávila, C. R .B., Consciência Fonológica. In: Ferreira, L. P., Befi-Lopes, D. M.; Limongi, S. C. O., (2004) Tratado de Fonoaudiologia (pp.815-824). São Paulo - Roca.

Blomert, L., Mitterer, H., \& Paffen, C. (2004). In Search of the Auditory, Phonetaic, and/or Phonological Problems in Dyslexia: Context Effects of Speech Perception. Journal of Speech, Language, and Hearing Research, 47, 1030-1047.

Boets, B., Wouters, J., Wieringen, J., \& Ghesquière, P. (2007). Auditory processing, speech perception and phonological ability in pre-school children at high-risk for dyslexia: A longitudinal study of the auditory temporal processing theory. Neuropsychologia, 45(8), 1608-1620.

Borges, A. C. L. C. (1997). Dissílabos Alternados SSW. Em L. D. Pereira \& E. Schochat (Orgs.),
Processamento auditivo central: Manual de avaliação (pp. 169-178). São Paulo: Lovise.

Bradley, L., \& Bryant, P. E. (1983). Categorising sounds and learning to read a causal connection. Nature, 301, 419-421.

Byrne, B., \& Fielding-Barnsley, R. (1989). Phonemic awareness and letter knowledge in the child's acquisition of the alphabetic principle. Journal of Educational Psychology, 81(3), 313-321.

Capellini, S. A. (2004). Distúrbios de aprendizagem versus dislexia. Em L. P. Ferreira, D. Befi-Lopes \& S. Limongi (Orgs.), Tratado de fonoaudiologia (pp. 862876). São Paulo: Roca.

Capellini, S. A., \& Cavalheiro, L. G. (2000). Avaliação do nível e da velocidade de leitura em escolares com e sem dificuldade na leitura. Temas sobre Desenvolvimento, 9(51), 5-12.

Capellini, S. A., Ferreira, T. L., Salgado, C. A., \& Ciasca, S. M. (2007). Desempenho de escolares bons leitores, com dislexia e com transtorno do déficit de atenção e hiperatividade em nomeação automática rápida. Jornal Brasileiro de Fonoaudiologia, 12, 114119.

Capellini, S. A., \& Oliveira, K. T. O. (2003). Problemas de aprendizagem relacionados às alterações de linguagem. Em S. M. Ciasca (Org.), Distúrbio de aprendizagem: proposta de avaliação interdisciplinar (pp. 113-140). São Paulo: Casa do Psicólogo.

Capellini, S. A., Padula, N. A. M. R., \& Ciasca, S. M. (2004). Desempenho de escolares com distúrbio específico de leitura em programa de remediação. PróFono, 16(3), 261-274.

Capovilla, A. G. S., \& Capovilla, F. C. (1998). Prova de consciência fonológica: desenvolvimento de dez 
habilidades da pré-escola à segunda série. Temas sobre desenvolvimento, 7(37), 14-20.

Cardoso, A. C. V. (1997). Perfil do processamento autiditivo em grupo de escolares do município de Marilia. Tese de Mestrado, Universidade Federal de São Paulo, São Paulo.

Cherry, R., \& Rubinstein, A. (2006). Comparing Monotic and Diotic Selective Auditory Attention Abilities in Children. Language, Speech and Hearing Services in Schools, 37, 137-142.

Ciasca, S. M. (1994). Distúrbios e dificuldades de aprendizagem em crianças: análise do diagnóstico interdisciplinar. Tese de Doutorado, Faculdade de Ciências Médicas, Universidade de Campinas, Campinas.

Critchley, M. (1985). Specific developmental dyslexia. Em J. A. M. Frederiks (Ed.), Handbook of neurology (pp. 491-506). Amsterdam: Elsevier.

Dally, K. (2006). The Influence of Phonological Processing and Inattentive Behavior on Reading Acquisition. Journal of Educational Psychology, 98(2), 420-437.

Dawes, P., Bishop, D. V. M., Sirimanna, T., \& Bamiou, D. E. (2008). Profile and a etiology of children diagnosed with auditory processing disorder (APD). Journal of Pediatric Otorhinolaryngology, 72, 483-489.

Doughterty, R. F., Cynader, M. S., Bjornson, B. H., Edgell, D., \& Giaschi, D. E. (1998). Dichotic pitch: a new stimulus distinguishes normal and dyslexic auditory function. Neuroreport, 9(13), 3001-3005.

Etchepareborda, M. C. (2002). Detección precoz de la dislexia y enfoque terapéutico. Revista de Neurología, 34(1), 13-23.
Frith, U. (1985). Beneath the surface of developmental dyslexia. Em K. E. Patterson, J. C. Marshall \& M. Coltheart (Eds.), Surface dyslexia: neuropsychological and cognitive analyses of phonological reading (pp. 301330). London: Lawrence Erlbaum.

Grégoire, J., \& Piérart, B. (1997). Avaliação dos problemas de leitura: os novos modelos teóricos e suas implicações diagnósticas. Porto Alegre: Artes Médicas.

Iliadou, V., \& Lakovides, S. (2003). Contribution of psychoacoustics and neuroaudiology in revealing correlation of mental disorders with central auditory processing disorders. Annals of General Hospital Psychiatry, 2, 1-5.

Jerger, J., Jerger, S., \& Hawkins, J. J. (1980). Pediatric Speech Intelligibility Test. Generation of test materials. http://www.sciencedirect.com/science?_ob=RedirectUR L\&_method=gejLink\&_linkType=general\&_cdi=5068\& _issn $=01655876 \&$ _targetURL $=$ http $\% 3 \mathrm{~A} \% 2 \mathrm{~F} \% 2 \mathrm{Fwww} . \mathrm{e}$ lsevier.com\%2Flocate $\% 2$ Fissn $\% 2$ F01655876\&_version= 1\&_userid=10\&md5=79ba430eefb6cefc 93 fbf 75 dd63e08 93 International Journal of Pediatric Otorhinolaryngology, 2, 217-30.

Kujala, T., Lovio, R., Lepisto, T., Laasonen, M., \& Näätänen, R. (2006). Evaluation of multi-attribute auditory discrimination in dyslexia with the mismatch negativity. Clinical Neurophysiology, 117, 885-893.

Lefreve, A. F. B. (1976). Exame neurológico evolutivo. São Paulo: Savier.

Liberman, I. Y., \& Shankweiler, D. (1989). Phonologie et apprentissage de la lecture: une introduction. Em C. A. Perfetti \& L. Rieben (Eds.), Laprrenti lecteur: reserches empiriques et implications pédagogiques (pp. 100-115). Paris: Delachaux et Niestle. 
López-Escribano, C. (2007). Contribuciones de la neurociencia al diagnóstico y tratamiento educativo de la dislexia del desarrollo. Revista de Neurologia, 44(3), 173-180.

Mendonça, M. P. C., \& Mendes, E. G. (2000). Efeito de um treino de habilidades fonológicas em crianças com dificuldades de leitura e escrita. Dissertação de Mestrado, Universidade Federal de São Carlos, São Carlos.

Moisescu-Yiflach, T., \& Pratt, H. (2005). Auditory event related potentials and source current density estimation in phonologic/auditory dyslexics. Clinical Neurophysiology, 115, 2632-2647.

Moraes, J. (1996). A arte de ler. São Paulo: Editora Unesp.

Musiek, F. E. (1999). Habilitation and Management of Auditory Processing Disorders: Overview of Selected Procedures. Journal of the American Academy of Audiology, 10, 329-342.

Olivares-García, M. R., Peñaloza-López, Y. R., GarcíaPedroza, F., Jesús-Pérez, S., Uribe-Escamilla, R., \& Jiménez, S. S. (2005). Identificación de la lateralidad auditiva mediante una prueba dicótica nueva con dígitos en español, y de la lateralidad corporal y orientación espacial en niños con dislexia y en controles. Revista de Neurología, 41(4), 198-205.

Olson, R. W., Wilse, B., Conners, F. A., \& Rack, J. P. (1990). Specific deficit in component reading and language skills: genetic and environmental influences. Journal of Learning Disabilities, 22, 339-348.

Ortiz, O., Jiménez, J. E., Miranda, E. G., Rosquete, R. G., Hernández-Valle, I., Rodrigo, M., Estévez, A., Diaz, A., \& Expósito, S. A. (2007). Locus and Nature of Perceptual Phonological Deficit in Spanish Children
With Reading Disabilities. Journal of Learning Disabilities, 40(1), 80-92.

Pereira, L. D. (1993). Processamento auditivo. Temas em Desenvolvimento, 2(11), 7-14.

Pereira, L. D. (1996). Identificação de desordem de processamento auditivo central através de observação comportamental: organização de procedimentos padronizados. Em E. Schochat (Org.), Processamento Auditivo (pp. 43-56). São Paulo: Lovise.

Pereira, L. D. (1997). Processamento auditivo central: abordagem passo a passo. Em L. D. Pereira \& E. Schochat (Orgs.), Processamento auditivo central: Manual de avaliação (pp. 49-59). São Paulo: Lovise.

Pereira, L. D., SchochaT, E. (1997) Processamento auditivo central: manual de avaliação (pp. 99 - 138). São Paulo: Lovise.

Pinheiro, A. M. V. (1994). Leitura e escrita: uma abordagem cognitiva. Campinas: Psy II.

Ratner, N. B., Gleason, J. B., \& Narasimhan, B. (1999). An introduction to psycholinguistics: what do language users know? Em J. B. Gleason \& N. B. Ratner (Eds.), Psycholinguistics (pp. 1-49). Philadelphia: Harcourt Brace College.

Santos, M. F. C., \& Pereira, L. D. (1997). Escuta com Dígitos. Em L. D. Pereira \& E. Schochat (Eds.), Processamento auditivo central: Manual de avaliação (pp. 147-149). São Paulo: Lovise.

Sauer, L. O. (2005). Processamento auditivo e SPECT em crianças com dislexia._Dissertação de mestrado, Universidade Estadual de Campinas, Campinas.

Sauer, L. O., Pereira, L. D., Ciasca, S. M., Pestun, M., \& Guerreiro, M. M. (2006). Processamento auditivo e 
SPECT em crianças com dislexia. Arquivos de Neuropsiquiatria, 64(1), 108-111.

Snowling, M. (1987). Dyslexia: a cognitive developmental perspective. Oxford: Blackwell.

Stanovich, K. E. (1988). Explaining the differences between the dyslexic and the garden-variety poor-reader; the phonological-core variable-difference model. Journal of Learning Disabilities, 21, 590-604.

Tallal, P., \& Gaab, N. (2008). Dynamic auditory processing, musical experience and language development. Trends in Neurosciences, 29(7), 382-90.

Tallal, P., Miller, S. L., Gail, B., Byma, G., Wang, X., Nagarajan, S. S., Schreiner, C., Jenkins, W. M., \&
Merzenich, M. M. (1996). Language Comprehension in Language-Learning Impaired Children Improved with Acoustically Modified Speech. Science, 271(5245), 8184.

Wechsler, D. (2002). Escala de inteligência para crianças (WISC III). São Paulo: Casa do Psicólogo.

Yavas, M. S., Hernandorena, C. L. M., \& Lamprecht, R. R. (1991). Avaliação fonológica da criança. Porto Alegre: Artes Médicas.

Zilioto, K. N., Kalil, D. M., \& Almeida, C. I. R. (1997). PSI em português. Em L. D. Pereira \& E. Schochat (Orgs.), Processamento auditivo central: Manual de avaliação (pp. 113-128). São Paulo: Lovise.

Recebido em: 16/10/2007

Revisado em: 13/06/2008

Aprovado em: 05/08/2008

Sobre as autoras:

Simone Aparecida Capellini (sacap@uol.com.br) - Fonoaudióloga. Doutora e Pós-Doutoranda em Ciências Médicas - Faculdade de Ciências Médicas da Universidade Estadual de Campinas - FCM/UNICAMP- Campinas - SP/Brasil. Docente do Departamento de Fonoaudiologia da Faculdade de Filosofia e Ciências da Universidade Estadual Paulista - FFC/UNESP-Marília - SP/Brasil.

Giseli Donadon Germano (giseliger@yahoo.com.br) - Fonoaudióloga. Mestranda no Programa de Pós-Graduação em Educação - Faculdade de Filosofia e Ciências da Universidade Estadual Paulista - FFC/UNESP-Marília - SP/Brasil.

Ana Cláudia Vieira Cardoso (acvjmc@uol.com.br) - Fonoaudióloga. Doutora em Distúrbios da Comunicação Humana - Universidade Federal de São Paulo- UNIFESP-EPM São Paulo - SP/ Brasil. Docente do Departamento de Fonoaudiologia da Faculdade de Filosofia e Ciências da Universidade Estadual Paulista - FFC/UNESP-Marília - SP/Brasil. 